\title{
Coloring and Guarding Arrangements
}

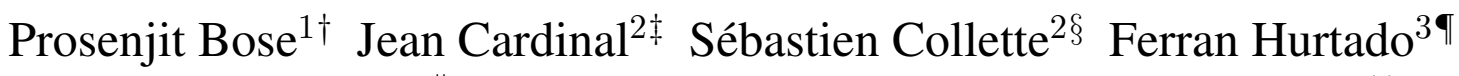 \\ Matias Korman $\left\|^{3}\right\| \quad$ Stefan Langerman $\|^{2}$ ** $\quad$ Perouz Taslakian $\|^{\dagger}$
}

${ }^{1}$ Carleton University, Ottawa, Canada.

${ }^{2}$ Université Libre de Bruxelles (ULB), Brussels, Belgium.

${ }^{3}$ Universitat Politècnica de Catalunya (UPC), Barcelona, Spain.

${ }^{4}$ American University of Armenia, Yerevan, Armenia.

received $22^{\text {nd }}$ Feb. 2012, revised 30th Sep. 2013, 12 th Nov. 2013, accepted $25^{\text {th }}$ Nov. 2013.

Given a simple arrangement of lines in the plane, what is the minimum number $c$ of colors required so that we can color all lines in a way that no cell of the arrangement is monochromatic? In this paper we give worst-case bounds on the number $c$ for both the above question and for some of its variations. Line coloring problems can be redefined as geometric hypergraph coloring problems as follows: if we define $\mathcal{H}_{\text {line-cell }}$ as the hypergraph whose vertices are lines and edges are cells of the arrangement, then $c$ is equal to the chromatic number of this hypergraph. Specifically, we prove that this chromatic number is between $\Omega(\log n / \log \log n)$ and $O(\sqrt{n})$.

Furthermore, we give bounds on the minimum size of a subset $S$ of the intersection points between pairs of lines in $\mathcal{A}$ such that every cell contains at least a vertex of $S$. This may be seen as the problem of guarding cells with vertices when the lines act as obstacles. The problem can also be defined as the minimum vertex cover problem in the hypergraph $\mathcal{H}_{\text {vertex-cell }}$, the vertices of which are the line intersections, and the hyperedges are vertices of a cell. Analogously, we consider the problem of touching the lines with a minimum subset of the cells of the arrangement, which we identify as the minimum vertex cover problem in the $\mathcal{H}_{c e l l-z o n e}$ hypergraph.

Keywords: line arrangement, hypergraph coloring, duality, independent set, vertex cover

\footnotetext{
${ }^{\dagger}$ Email: jitescs.carleton.ca.

¥Email: jcardineulb.ac.be. Partially supported by the the ESF EUROCORES programme EuroGIGA, CRP ComPoSe: Fonds National de la Recherche Scientique (F.R.S.-FNRS) - EUROGIGA NR 13604 for Belgium.

§Email: secollet@ulb.ac.be.

TEmail: ferran.hurtado@upc.edu. Partially supported by projects MINECO MTM2012-30951, Gen. Cat. DGR2009SGR1040, and ESF EUROCORES programme EuroGIGA - CRP 'ComPoSe': MICINN Project EUI-EURC-2011-4306.

IIEmail: matias.korman@upc.edu. Partially supported by projects MINECO MTM2012-30951, Gen. Cat. DGR2009SGR1040, ESF EUROCORES programme EuroGIGA - CRP 'ComPoSe’: MICINN Project EUI-EURC-2011-4306, and the Secretary for Universities and Research of the Ministry of Economy and Knowledge of the Government of Catalonia and the European Union.

${ }^{* *}$ Email: slanger@ulb.ac.be. Directeur de Recherches du F.R.S.-FNRS. Partially supported by the the ESF EUROCORES programme EuroGIGA, CRP ComPoSe: Fonds National de la Recherche Scientique (F.R.S.-FNRS) - EUROGIGA NR 13604 for Belgium.

$\dagger^{\dagger}$ Email: ptaslakian@aua.am.
}

1365-8050 @ 2013 Discrete Mathematics and Theoretical Computer Science (DMTCS), Nancy, France 


\section{Introduction}

While dual transformations may allow converting a combinatorial geometry problem about a configuration of points into a problem about an arrangement of lines the truth is that most mathematical questions appear to be much cleaner and natural in only one of the settings. In many cases, the dual version is considered solely when, rather than making sense, it is additionally useful. Both kinds of geometric objects have inspired many problems and attracted much attention. For finite point sets, the Erdôs-Szekeres problem on finding large subsets in convex position, or the repeated distances problem on how many times a single distance can appear between pairs of points, are examples of famous questions that have been pursued for decades and are still open. Many research problems of this kind are described in [3], Chapter 8. Concerning arrangements of lines, possibly the most prevalent problems consist of studying the number of cells of each size, say triangles, that appear in every arrangement; however, many other questions have also been considered (see [4, 7, 8|). There are also problems that combine both kinds of objects, like counting incidences between points and lines, or studying the arrangements of lines spanned by point sets, which includes the celebrated Sylvester-Gallai problem [3] on ordinary lines (containing exactly two input points).

In the first class of problems, substantial attention has been given to colored point sets (that is, configurations of points that belong to several classes or colors). Among those, we highlight the chromatic variants of the repeated distances problem and the Erdős-Szekeres problem, and colored versions of Tverberg's Theorem and Helly's Theorem. In particular there is a vast body of research on problems involving a set of red points and a set of blue points. Refer to [12] for a survey on red-blue problems, or to [3] for a more generic account.

Somehow surprisingly, there does not exist a comparable set of questions that have been studied for colored arrangements of lines. There is a series of papers on bicolored sets of lines that study the existence of monochromatic vertices [9. 10, 15] (intersection points of lines of the same color). Another series of papers study colorings of the so-called arrangement graphs, in which vertices are the intersection points and edges are the segments between any two that are consecutive on one of the lines [2,5].

However, many other natural questions can be asked. For example, is it true that every bicolored arrangement of lines has a monochromatic cell? We prove in this paper that the generic answer is "no", but that it is "yes" when the colors are slightly unbalanced. This leads immediately to another question that we discuss: How many colors are always sufficient, and occasionally necessary, to color any set of $n$ lines in such a way that the induced arrangement contains no monochromatic cell?

The last question is reminiscent of Art Gallery Problems [16-18], where we consider several questions that, to our knowledge, have not been studied before in the context of line arrangements. Examples of such questions that we consider are: How many vertices of an arrangement suffice to guard (touch) all of its cells? How many lines are enough to guard all cells?

While at first glance the problem of coloring arrangements of lines may appear unrelated to that of guarding, there is a clean unifying framework provided by considering appropriate geometric hypergraphs. For example, minimally coloring an arrangement while avoiding monochromatic cells can be reformulated as finding the chromatic number of the hypergraph $\mathcal{H}_{\text {line-cell }}$, whose vertices are lines, and edges represent cells of the arrangement. Guarding all cells with a small number of lines can be seen as a vertex cover of the same hypergraph $\mathcal{H}_{\text {line-cell }}$.

In this work we consider several questions that are variations on the themes of coloring and guarding arrangements of lines, which translate consistently into problems on geometric hypergraphs, such as size 
of maximal independent set, vertex cover, or chromatic number.

The terminology for hypergraphs on arrangements is introduced in Section 2 , where we also provide a table summarizing our results. Coloring problems are then discussed in Section 3 and guarding problems in Section 4 . We conclude with some observations and open problems.

\section{Definitions and Summary of Results}

Let $\mathcal{A}$ be an arrangement of a set of lines $L$ in $\mathbb{R}^{2}$. We say that an arrangement of lines $\mathcal{A}$ is simple if every two lines intersect, and no three lines have a common intersection point. From now on, we only consider simple arrangements of lines (i)

Any arrangement $\mathcal{A}$ decomposes the plane into different cells, where a cell is a maximal connected component of $\mathbb{R}^{2} \backslash L$. We define $\mathcal{H}_{\text {line-cell }}=(L, C)$ as the geometric hypergraph corresponding to the arrangement, where $C$ is the set containing all cells of $\mathcal{A}$. Similarly, $\mathcal{H}_{\text {vertex-cell }}=(V, C)$ is the hypergraph defined by the vertices of the arrangements and its cells, where $V=\left(\begin{array}{c}L \\ 2\end{array}\right)$ is the set of intersection of lines in $\mathcal{A}$. Finally, $\mathcal{H}_{\text {cell-zone }}=(C, Z)$ is the hypergraph defined by the cells of the arrangement and its zones. The zone of a line $\ell$ in $\mathcal{A}$ is the set of cells bounded by $\ell$. The set $Z$ is defined as the set of subsets of $C$ induced by the zones of $\mathcal{A}$. Note that this hypergraph is the dual hypergraph of $\mathcal{H}_{\text {line-cell }}$.

An independent set of a hypergraph $\mathcal{H}=(S, E)$ is a set $S^{\prime} \subseteq S$ such that $\forall e \in E: e \not \subseteq S^{\prime}$. This definition is the natural extension from the graph variant, and requires that no hyperedge is completely contained in $S^{\prime}$. Analogously, a vertex cover of $\mathcal{H}$ is a set $S^{\prime} \subseteq S$ such that $\forall e \in E: e \cap S^{\prime} \neq \emptyset$. The chromatic number $\chi(\mathcal{H})$ of $\mathcal{H}$ is the minimum number of colors that can be assigned to the vertices $v \in S$ so that no hyperedge containing two or more vertices is monochromatic (that is, $\forall e \in E$ such that $|e|>1$, there exist $v_{1}, v_{2} \in e$ such that $\left.\operatorname{col}\left(v_{1}\right) \neq \operatorname{col}\left(v_{2}\right)\right)$.

In the forthcoming sections we give upper and lower bounds on the worst-case values for these quantities on the three hypergraphs defined on a given line arrangement. Our results are summarized in Table 1. Note that the maximum independent set and minimum vertex cover problems are complementary. As a result, any lower bound on one gives an upper bound on the other and vice versa. This property, along with the facts that $|L|=n,|V|=\left(\begin{array}{l}n \\ 2\end{array}\right)$, and $|C|=\frac{n(n+1)}{2}+1$, are used to complement many entries of the table.

The definitions of an independent set and a proper coloring of the $\mathcal{H}_{\text {line-cell }}$ hypergraph of an arrangement are illustrated in Figures 1(a) and 1(b), respectively. Similarly, the definition of a vertex cover of the $\mathcal{H}_{\text {vertex-cell }}$ and $\mathcal{H}_{\text {cell-zone }}$ hypergraphs are illustrated in Figures 1(c), and 1(d), respectively.

\begin{tabular}{|c|c|c|c|}
\hline Hypergraph & Max. Ind. Set & Vertex Cover & Chromatic number \\
\hline $\mathcal{H}_{\text {line-cell }}$ & $\begin{array}{l}\geq \sqrt{n+1}-1 \text { (Th. } 3) \\
\leq \frac{2 n}{3} \text { (Th. } 4\end{array}$ & $\begin{array}{c}\left.\geq \frac{n}{3} \text { (Cor. } 14\right) \\
\leq n+1-\sqrt{n+1} \text { Cor. } 14)\end{array}$ & $\begin{array}{l}\Omega(\log n / \log \log n)(\text { Th. } \\
\quad \leq 2 \sqrt{n}+O(1)(\text { Th. } 5)\end{array}$ \\
\hline $\mathcal{H}_{\text {vertex }- \text { cell }}$ & $\begin{array}{l}\geq \frac{n^{2}}{3}-\frac{5 n}{2} \text { (Lem. } 10 \\
\leq \frac{n^{2}}{3}-\frac{n}{2} \text { (Lem. } 10\end{array}$ & $\begin{aligned} & \geq \frac{n^{2}}{6} \text { (Th. 9) } \\
\leq & \left.\frac{n^{2}}{6}+n \text { (Th. } 9\right)\end{aligned}$ & $\begin{array}{l}\geq 2(\text { Trivial }) \\
\leq 3(\text { Obs. } 1\end{array}$ \\
\hline $\mathcal{H}_{\text {cell-zone }}$ & $\begin{array}{l}\left.\geq \frac{n^{2}}{2}+\frac{5 n}{48}-o(n) \text { (Cor. } 13\right) \\
\left.\leq \frac{n^{2}}{2}+\frac{5 n}{4}+1 \text { (Cor. } 13\right)\end{array}$ & $\begin{array}{c}\geq \frac{n}{4}(\mathrm{Th} .12 \\
\leq \frac{19 n}{48}+o(n)(\text { Th. } 12\end{array}$ & 2 (Th. 8 \\
\hline
\end{tabular}

Tab. 1: Summary of worst-case bounds for the different problems studied in this paper.

(i) For non-simple arrangements, the answer to most of the problems we study are either trivial or not well defined. 


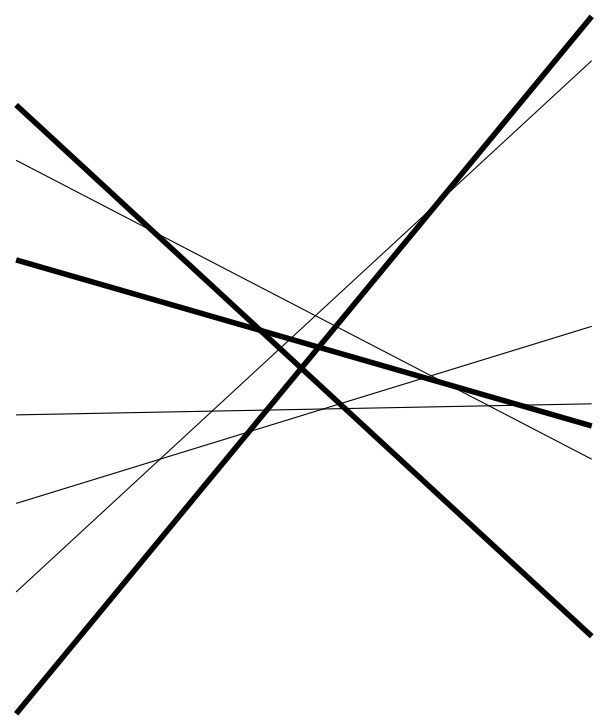

(a) The thick lines form an independent set in the $\mathcal{H}_{\text {line-cell }}$ hypergraph: no cell is bounded by these lines only.

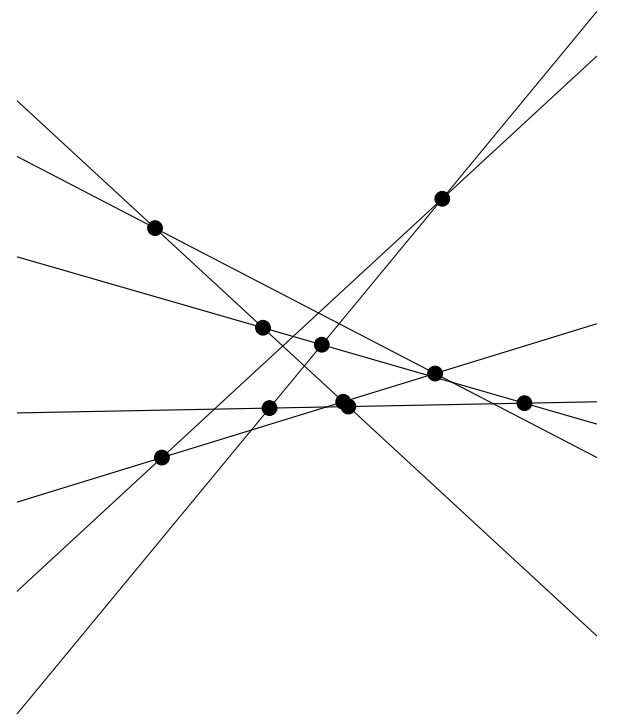

(c) The marked intersections form a vertex cover of the $\mathcal{H}_{\text {vertex-cell }}$ hypergraph: every cell has at least one such intersection on its boundary. That is, these vertices guard the cells.

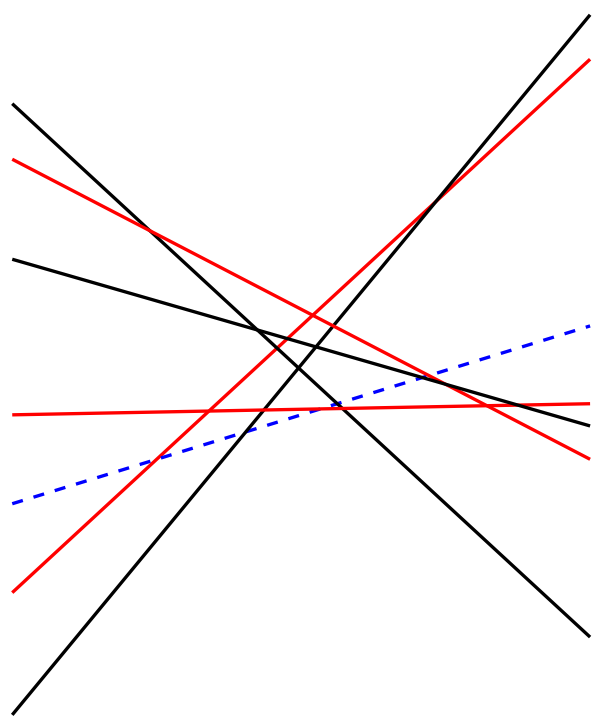

(b) A proper 3-coloring of the $\mathcal{H}_{\text {line-cell }}$ hypergraph: no cell is monochromatic.

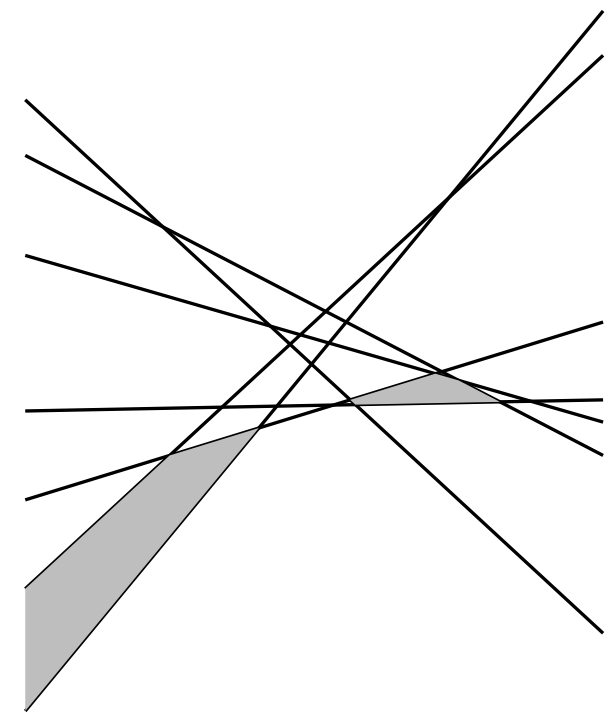

(d) The two marked cells form a vertex cover of the $\mathcal{H}_{\text {cell-zone }}$ hypergraph: every line has a segment that lies on the boundary of one of these cells. That is, these two cells guard the lines.

Fig. 1: Illustrations of the definitions. 


\section{Coloring Lines, and Related Results}

We first consider the chromatic number of the line-cell hypergraph of an arrangement, that is, the number of colors required for coloring the lines so that no cell has a monochromatic boundary. At the end of the section we include other results for similar hypergraphs.

\subsection{Two-colorability}

We say that a set of lines $L$ is $k$-colorable if we can color $L$ with $k$ colors such that no cell is monochromatic (in other words, the corresponding $\mathcal{H}_{\text {line-cell }}$ hypergraph has chromatic number at most $k$ ). Any coloring $c: L \rightarrow\{0, \ldots, k\}$ that satisfies such a property is said to be proper. We first tackle the (simple) question of whether the two-colorable $\mathcal{H}_{\text {line-cell }}$ hypergraphs have bounded size.

Theorem 1 There are arbitrarily large two-colorable sets of lines.

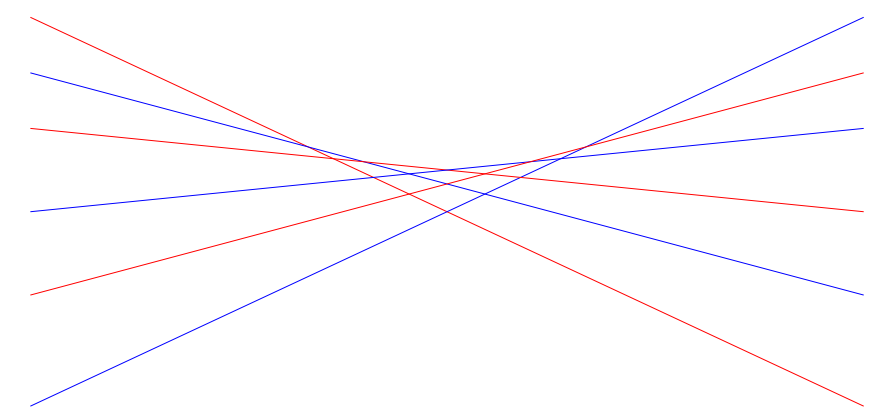

Fig. 2: A set of lines that can be colored with two colors without a monochromatic cell.

Proof: For any $q \in \mathbb{N}$ consider a set of $2 q$ lines in convex position (a set of lines whose projective dual is a set of points in convex position, see Figure 2). In such an arrangement of lines, each cell is bounded by either (1) two consecutive lines, (2) the first and the last line or (3) all lines of the arrangement. Thus, if we color the lines alternatingly red and blue by order of slope, no cell will be monochromatic.

The coloring used in Theorem 1 uses roughly the same number of lines of each color. This is not a coincidence, since we now show that any proper coloring must satisfy a similar property.

Theorem 2 Each color class of a proper two-coloring $L \rightarrow\{0,1\}$ of a set $L$ of $n$ lines has size at most $\frac{n}{2}+\frac{\sqrt{n-1}-1}{2}$.

Proof: Let $R$ be the set of lines that are assigned color 0 , and $B$ the set of lines that are assigned color 1 . Let $\mathcal{A}_{R}$ denote the arrangement of the lines in $R$. As $R$ does not completely define a cell of $\mathcal{A}$, each cell of $\mathcal{A}_{R}$ must be traversed by a line in $B$.

We proceed iteratively: we start with $\mathcal{A}_{R}$, and add the lines of $B$ one at a time. When adding a line $\ell$, some cells of $\mathcal{A}_{R}$ will be split into two by a line segment induced by $\ell$.

To each cell $c$ of $\mathcal{A}_{R}$, we assign a number that represents the number of connected components defined by the segments inside $c$ (that is, segments whose intersection graph is connected). Let $f(c, i)$ denote the 
number of connected components inside the cell $c$ after adding the $i$ - th line of $B$, and $f\left(\mathcal{A}_{R}, i\right)$ is the sum of $f(c, i)$ over all cells $c \in \mathcal{A}_{R}$.

When the first line $\ell_{1} \in B$ is added, $f(c, 1)=1$ for each cell $c$ crossed by $\ell_{1}$, and remains zero for every other cell. Since $\ell_{1}$ will cross all lines of $R$ exactly once, we have $f\left(\mathcal{A}_{R}, 1\right)=|R|+1$. In general, when the $i-$ th line $\ell_{i}$ is added, $f\left(\mathcal{A}_{R}, i\right)$ increases by $|R|+2-i$. Indeed, in each cell $c$, the blue line can only intersect each component once; otherwise the corresponding segments would create a cycle, meaning that a new face bounded only by blue lines (and thus monochromatic) is created. This implies that $\ell_{i}$ intersects all previous $i-1$ lines in $i-1$ disjoint components. Inside a cell $c$, if a line $\ell_{i}$ intersects $t$ components, then $f(c, i)=f(c, i-1)-t+1$. Thus, $f\left(\mathcal{A}_{R}, i\right)=f\left(\mathcal{A}_{R}, i-1\right)-(i-1)+|R|+1=f\left(\mathcal{A}_{R}, i-1\right)+|R|+2-i$.

What we also know is that at the end of this process, each cell of $\mathcal{A}_{R}$ contains at least one component, otherwise the cell is monochromatic. Thus $f\left(\mathcal{A}_{R},|B|\right)$ is bigger than or equal to the number of cells in $\mathcal{A}_{R}$.

We get:

$$
\begin{aligned}
\frac{|R| \cdot(|R|+1)}{2}+1 & \leq f\left(\mathcal{A}_{R},|B|\right)=\sum_{i=1}^{|B|}|R|+2-i, \\
|R|(|R|+1)+2 & \leq 2|B| \cdot(|R|+2)-2 \sum_{i=1}^{|B|} i \\
|R|^{2}+|R|+2 & \leq 2|B| \cdot(|R|+2)-|B| \cdot(|B|+1) \\
|R|^{2}+|R|+2 & \leq 2(n-|R|) \cdot(|R|+2)-(n-|R|) \cdot(n-|R|+1) \\
|R|^{2}+|R|+2 & \leq 2\left(-|R|^{2}+(n-2)|R|+2 n\right)-\left(|R|^{2}-(1+2 n)|R|+\left(n^{2}+n\right)\right) \\
4|R|^{2}+4(1-n)|R|+n^{2}-3 n+2 & \leq 0 \\
|R| & \leq \frac{4(n-1)+\sqrt{16(1-n)^{2}-16\left(n^{2}-3 n+2\right)}}{8} \\
|R| & \leq \frac{(n-1)+\sqrt{n-1}}{2}=\frac{n}{2}+\frac{\sqrt{n-1}-1}{2}
\end{aligned}
$$

which concludes the proof.

\subsection{Independent lines in $\mathcal{H}_{\text {line-cell }}$}

Recall that an independent set of lines in an arrangement is defined as a subset of lines $S$ so that no cell of the arrangement is only adjacent to lines in $S$.

Theorem 3 For any set $L$ of $n$ lines, the corresponding $\mathcal{H}_{\text {line-cell }}$ hypergraph has an independent set of size $\sqrt{n+1}-1$.

Proof: We prove that any (inclusionwise) maximal independent set has size $\sqrt{n+1}-1$. Consider such a maximal independent set $I \subset L$. By maximality, each line $\ell \in L \backslash I$ can be associated with at least one cell of $L$ whose boundary consists only of one segment of $\ell$, and segments of lines in $I$ (otherwise we could add $\ell$ to $I$, resulting in a contradiction). For each line $\ell \in L \backslash I$ we pick one such cell (arbitrarily). We call this cell the witness of $\ell$ and denote it by $c_{\ell}$. 
In order to prove our claim, we use the following charging scheme for all lines of $\ell$ : if $c_{\ell}$ is formed by exactly two lines, we charge $\ell$ to the only line of $I$ in $c_{\ell}$. Otherwise, $c_{\ell}$ is defined by many lines and all $\ell$ belong to $I$. In particular, it must contain a vertex defined by the intersection of two lines of $I$. We charge $\ell$ to the first such vertex that we encounter when we traverse the boundary of $c_{\ell}$ starting from the segment defined by $\ell$ and continuing in a clockwise fashion. We note that lines of $I$ and the intersection point between two lines of $I$ can only be charged twice (a line can only belong to two cells of size two. Likewise an intersection point appears in four different cells, but it cannot be charged to two adjacent cells). Thus, we obtain the following bound on the size of $I$ :

$$
\begin{aligned}
|L \backslash I| & \leq 2\left(\begin{array}{c}
|I| \\
2
\end{array}\right)+2|I|, \\
n-|I| & \leq|I|(|I|-1)+2|I|, \\
0 & \leq|I|^{2}+2|I|-n, \\
|I| & \geq \sqrt{n+1}-1
\end{aligned}
$$

Theorem 4 Given a set $L$ of $n$ lines, an independent set of the corresponding $\mathcal{H}_{\text {line-cell }}$ hypergraph has size at most $2 n / 3$.

Proof: Let $S$ be an independent set of lines in $\mathcal{H}_{\text {line-cell }}$. This means that, in the corresponding arrangement $\mathcal{A}_{S}$, each cell is touched by at least a line $\ell \in L \backslash S$ (otherwise it would not be independent). Each line $\ell \in L \backslash S$ crosses $|S|+1$ cells of $\mathcal{A}_{S}$. There are $\frac{|S| \cdot(|S|+1)}{2}+1$ cells in $\mathcal{A}_{S}$, and thus

$$
\begin{gathered}
|L \backslash S| \cdot(|S|+1)=(n-|S|) \cdot(|S|+1) \geq \frac{|S| \cdot(|S|+1)}{2+1} \\
n \geq \frac{3|S|}{2}+\frac{1}{|S|+1}>\frac{3|S|}{2} \Rightarrow|S|<\frac{2 n}{3}
\end{gathered}
$$

\subsection{Chromatic number of $\mathcal{H}_{\text {line-cell }}$}

In this section, we study the problem of coloring the $\mathcal{H}_{\text {line-cell }}$ hypergraph. That is, we want to color the set $L$ so that no cell is monochromatic. We start by giving an upper bound on the required number of colors.

Theorem 5 Any arrangement of $n$ lines can be colored with at most $2 \sqrt{n}+O(1)$ colors so that no edge of the associated $\mathcal{H}_{\text {line-cell }}$ hypergraph is monochromatic.

Before giving the proof we note that, following a preliminary publication of this research, Ackerman and Pinchasi [1] improved the upper bound by showing that $O(\sqrt{n / \log n})$ colors are sufficient to color the $\mathcal{H}_{\text {line-cell }}$ hypergraph. In their work, they also relate this problem with a longstanding open problem by Erdős : Estimating the maximum number of points in general position within a set of $n$ points that has no more than three collinear points. For completeness, we present a proof of Theorem 5 . 
Proof: Our coloring scheme is as follows: select the largest independent set $I$, color all the lines of $I$ with the same color, remove $I$ from $L$, and iterate this process on the remaining lines. At each step of the algorithm, we assign a different color to the lines we remove. The algorithm stops whenever the number of non-colored lines is at most $n_{0}$ (where $n_{0}$ is the bound from Theorem 3). Whenever $n_{0}$ or fewer lines remain, we complete the coloring by adding a new color to each of the remaining lines.

First we show that this method indeed provides a proper coloring. For this purpose, observe that any independent set of $L^{\prime} \subseteq L$ is also an independent set of $L$. That is, lines with the same color form an independent set of $L$. In particular, there cannot exist a cell whose associated lines are assigned the same color.

Let $c(n)$ be the maximum number of colors used for an arrangement of $n$ lines with the above coloring strategy. We claim that for any $\varepsilon>0$ and for any sufficiently large $n, c(n) \leq(2+\varepsilon) \sqrt{n}+O(1)$ (note that this implies $c(n) \leq 2 \sqrt{n}+O(1)$ ).

Recall that, by Theorem 3 , the size of a maximal independent set is at least $\sqrt{n+1}-1$. Thus, we obtain the following recursion:

$$
c(n) \leq c(n-\sqrt{n+1}-1)+1
$$

We now assume that $c(n) \leq k \sqrt{n}$ for some constant $k$. By substituting in the previous inequality we obtain:

$$
c(n) \leq k \sqrt{n+1-\sqrt{n+1}}+1
$$

Thus, in order to complete the proof we must show that for any $k>2$ the following holds:

$$
\begin{aligned}
\sqrt{n+1-\sqrt{n+1}+1 / k} & \leq \sqrt{n} \Leftrightarrow \\
k & \leq \frac{1}{\sqrt{n}-\sqrt{n+1-\sqrt{n+1}}}
\end{aligned}
$$

The limit of this ratio is 2 , thus the claim holds for any constant larger than 2 as desired.

We now construct a slightly sublogarithmic lower bound for the chromatic number of $\mathcal{H}_{\text {line-cell }}$.

Theorem 6 For any $n_{0} \in \mathbb{N}$, there exists an arrangement of $n \geq n_{0}$ lines whose corresponding hypergraph $\mathcal{H}_{\text {line-cell }}$ has chromatic number $\Omega(\log n / \log \log n)$.

In the following we construct a set of roughly $k^{k}$ lines in which any $k$-coloring will induce a monochromatic cell (for any $k>0$ ). Since we are interested in the asymptotic behavior, it suffices to consider the case in which $k+1=2^{q}$ for some $q \geq 0$. In order to proceed with the proof, we first introduce some definitions and helpful results.

For any $x_{0} \in \mathbb{R}$ we consider the order of the intersections of the lines of $L$ with the vertical line of equation $x=x_{0}$, from top to bottom. Although the permutation obtained will depend on $L$ and $x_{0}$, there will be exactly $\left(\begin{array}{l}n \\ 2\end{array}\right)$ different permutations in any set $L$ of $n$ lines. Let $\Pi_{L}$ be the set of the different permutations that we can obtain on a line arrangement $L$. Each of these permutations is called a snapshot of $L$. We say that a real number $x_{0}$ realizes a snapshot $\pi$ if the ordering in the line $x=x_{0}$ is $\pi$. 

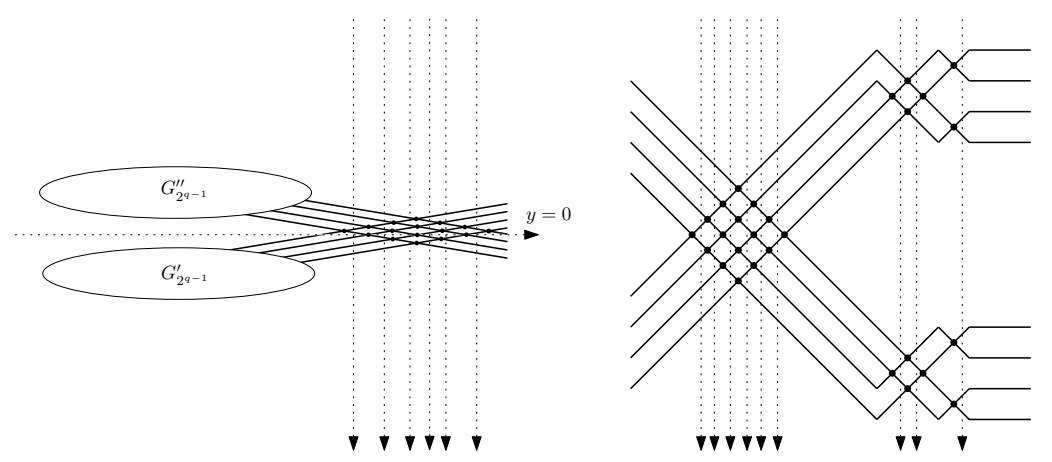

Fig. 3: Induction step in the gadget $G_{2^{q}}$ construction (left). The additional snapshots are shown as dashed vertical lines. The generated arrangement for $q=8$ and its witness set is shown on the right. For clarity, lines of $G_{8}$ have been depicted as pseudolines.

We say that a set of snapshots $\mathcal{W} \subseteq \Pi_{L}$ is a witness set of $L$ if for any two distinct lines $\ell, \ell^{\prime} \in L$, there exists a snapshot $\pi \in \mathcal{W}$ in which the two lines appear consecutively in $\pi$. It is easy to see that the entire set $\Pi_{L}$ is a witness set of quadratic size for any set of lines. Since the size of the witness set has a direct impact on our bound, we first show how to construct a line arrangement with a small witness set.

Lemma 7 For any $q \geq 0$ there exists a set $L$ of $2^{q}$ lines and a witness set $\mathcal{W}$ of $L$ such that $|\mathcal{W}| \leq 2^{q+1}$.

Proof: We construct the arrangement by induction on $q$. For $q=0$ our base gadget $G_{1}$ consists of a single line. Note that the witness property is vacuous, hence we define $\mathcal{W}_{1}=\emptyset$.

As we are only interested in the ordering in which lines are crossed, we can do any linear transformation to a set $L$ of lines, provided that transformation preserves the permutations in the set $\Pi_{L}$. If we update the coordinates of the snapshots in the witness set accordingly, the witness property still holds. In particular, we can transform a set $L$ of lines so that their slopes lie in an arbitrary small interval. We call this operation the flattening of $L$.

With this operation in mind we can do the induction step as follows: for any $q>0$ generate a copy of gadget $G_{2^{q-1}}$ and flatten the lines so that they all have small positive slopes and all crossings between any two lines occur below the horizontal line $y=0$. Let $G_{2^{q-1}}^{\prime}$ be the transformed set of lines and $G_{2 q-1}^{\prime \prime}$ be the reflexion of $G_{2^{q-1}}^{\prime}$ with respect to line $y=0$. The gadget $G_{2^{q}}$ is defined as the union of $G_{2^{q-1}}^{\prime}$ and $G_{2^{q-1}}^{\prime \prime}$ (see Figure 3 .

$G_{2^{q}}$ satisfies the following properties:

(i) $G_{2^{q}}$ has size exactly $2^{q}$. Moreover, any two lines cross exactly once.

(ii) The witness set $\mathcal{W}_{2^{q-1}}$ of $G_{2^{q-1}}^{\prime}$ also acts as witness set of $G_{2^{q-1}}^{\prime \prime}$.

(iii) The lines of $G_{2^{q-1}}^{\prime}$ and $G_{2^{q-1}}^{\prime \prime}$ intersect in a grid-like fashion, forming cells of size 4 and 2.

Observe that property $(i)$ certifies that the construction is a valid set of lines, while properties $(i i)$ and (iii) help us obtain a witness set $\mathcal{W}_{2^{q}}$ of small size; the crossing between lines of different gadgets can be guarded with $n-2=2^{q}-2$ snapshots (see Figure 3). Moreover, the crossings between lines of the same 
gadget can be guarded by the witness set $\mathcal{W}_{2^{q-1}}$ (which by induction satisfies $\left|\mathcal{W}_{2^{q-1}}\right| \leq 2 \cdot 2^{q-1}=2^{q}$ ). By construction we have that $\left|\mathcal{W}_{2^{q}}\right|=\left(2^{q}-2\right)+\left|\mathcal{W}_{2^{q-1}}\right|<2^{q}+2^{q}=2^{q+1}$.

To complete the proof we must show that $\mathcal{W}_{2^{q}}$ is indeed a witness set of $L$ : Let $\ell, \ell^{\prime}$ be any two lines of $L$. If these lines belong to the same sub-gadget, we can apply induction and obtain that they must be consecutive in one of the first snapshots. Otherwise, the two lines belong to different sub-gadgets of $G_{2^{q}}$, hence they will be consecutive at the latter snapshots.

With the previous result we can now prove Theorem 6 .

Proof: Let $L^{(0)}$ be the set of lines constructed in Lemma 7 and let $\mathcal{W}^{(0)}$ be the witness set of $L^{(0)}$ (recall that we have $\left|L^{(0)}\right|=k+1=2^{q}$ and $\left|\mathcal{W}^{(0)}\right|=m$ for some $m \leq 2(k+1)$ ). In general, a witness set $\mathcal{W}$ contains several snapshots. We number these snapshots from left to right. That is, we have $\mathcal{W}=\left\{\pi_{1}, \ldots, \pi_{m}\right\}$, and $\pi_{1}$ is the snapshot whose realizer has smallest $x$-coordinate. We note that this ordering is well defined, since it does not depend on the choice of the realizer.

Consider now any coloring of $L^{(0)}$ with $k$ colors. By the pigeonhole principle, there must exist two lines $\ell, \ell^{\prime}$ with the same assigned color. Since $\mathcal{W}^{(0)}$ is a witness set, these two lines must be consecutive at some snapshot $\pi \in \mathcal{W}^{(0)}$. Whenever this happens, we say that $\ell$ and $\ell^{\prime}$ form a monochromatic consecutive pair at snapshot $\pi$.

With the above definitions we can give an intuitive idea of our construction. Our goal is to cross two monochromatic consecutive pairs that are colored with the same color, thereby obtaining a monochromatic quadrilateral. The main difficulty of the proof is that we do not know which snapshot contains the monochromatic consecutive pair, nor which color it is assigned. In order to reduce the size of the witness set we essentially cross $k+1$ copies of $\mathcal{W}^{(0)}$. The main property of the construction is that these copies pairwise cross at the first snapshot. Thus, if all copies have their monochromatic consecutive pair at the first (that is, leftmost) snapshot, we can use the pigeonhole principle and obtain a monochromatic quadrilateral. Otherwise, at least one of the copies has a monochromatic consecutive pair at another snapshot, and in particular we can ignore the first snapshot of $\mathcal{W}^{(0)}$. That is, by making $k+1$ copies of the original line arrangement we can reduce the size of the witness set by one. If we repeat this process sufficiently many times, we obtain a line arrangement whose witness set has a single snapshot. We complete the construction by crossing $k+1$ copies of such gadget to finally obtain the desired monochromatic quadrilateral.

In general we use a set $L^{(i)}$ containing $(k+1)^{i+1}$ lines (for any $i \in\{0, \ldots, m+1\}$ ). In any $k$ coloring of the set $L^{(i)}$, our objective is to have either a monochromatic cell, or two lines that form a monochromatic consecutive pair at some snapshot $\pi_{j}$ (for $i \leq j \leq m$ ). In particular, the second situation cannot occur for set $L^{(m+1)}$, hence it must contain a monochromatic cell.

We now specify how to construct the set $L^{(i)}$ by induction on $i$. By flattening the lines of $L^{(0)}$ and scaling the instance, we can ensure that, for any $i \leq m$, the permutation induced by the line of equation $x=i$ is equal to the $i$ th snapshot of $\mathcal{W}^{(0)}$ (that is, the $x$-coordinate $i$ realizes the $i$ th snapshot of $\mathcal{W}^{(0)}$ ). For any $i>0$, our aim is to construct $L^{(i)}$ with $k+1$ different copies of set $L^{(i-1)}$ flattened and scaled so that they satisfy the following properties:

(i) For any $j \in\{i, \ldots m\}$, the ordering of each of the copies of $L^{(i-1)}$ at line $x=j$ corresponds to the $j$ th snapshot of $\mathcal{W}^{(i-1)}$. That is, $j$ realizes the $j$ th snapshot of each copy $L^{(i-1)}$.

(ii) No two lines of the same copy of $L^{(i-1)}$ cross in the vertical strip $\{-m<x<i\}$. In particular, the snapshot taken at any coordinate of the strip is $\pi_{i}$. 


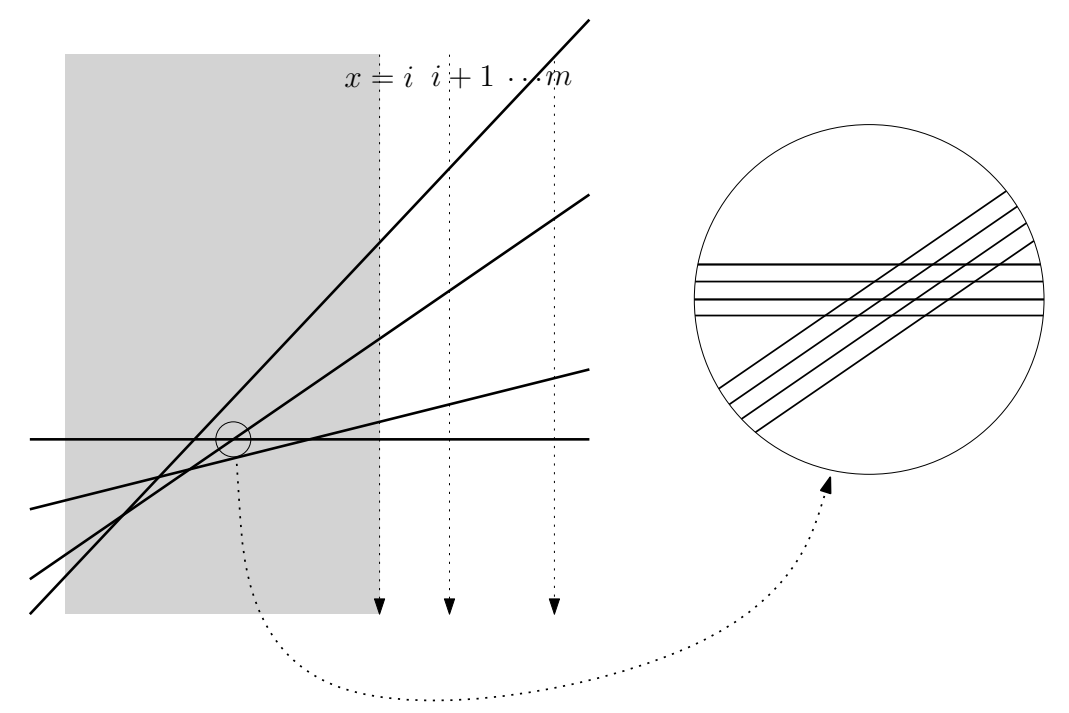

Fig. 4: Construction of the set $L^{(i)}$ by combining $k+1$ different copies of $L^{(i-1)}$. In the Figure, each thick line represents a copy of $L^{(i-1)}$ (and the dotted vertical lines the snapshots of $\pi_{j}$, for $j \geq i$ ). Observe that all the crossings between lines of different copies occur in the vertical strip $\{0<x<i\}$ (depicted as light gray in the figure). Since no crossing between lines of the same copy occur in the strip, the vertical ordering of the lines of a single copy at any position in the strip is exactly $\pi_{i}$. In the right we depict a larger image of the crossing between two copies of $L^{(i-1)}$. The main property is that any two consecutive lines of a copy of $L^{(i-1)}$ form a quadrilateral with any other pair of consecutive lines of another copy of $L^{(i-1)}$.

(iii) Lines of two different copies of $L^{(i-1)}$ cross in the vertical strip $\{0<x<i\}$ in a grid-like fashion. In particular, any two lines that are consecutive in $\pi_{i}$ form a quadrilateral with other two consecutive lines of another copy of $L^{(i-1)}$.

Such a construction can be obtained by flattening all the copies of $L^{(i-1)}$, placing the different copies in convex position, and scaling the instance (see Figure 4). Observe that since $L^{(i)}$ is composed of $k+1$ different copies of $L^{(i-1)}$, we indeed have $\left|L^{(i)}\right|=(k+1)\left|L^{(i-1)}\right|=(k+1)^{i+1}$.

We define the witness set $\mathcal{W}^{(i)}$ of $L^{(i)}$ as follows. For any $j \in\{i+1, \ldots, m\}$ we consider the line $x=j$, and add the corresponding snapshot to $\mathcal{W}^{(i)}$. By definition, we have $\left|\mathcal{W}^{(i)}\right|=m-i$. Since the copies do not cross at those coordinates, the $j$ th snapshot of $\mathcal{W}^{(i)}$ essentially consists of $k+1$ copies of the $j$ th snapshot of $\mathcal{W}^{(i-1)}$ one after the other.

In order to complete the proof we must show that in any coloring $c$ of $L^{(i)}$, we either have a monochromatic cell or a monochromatic consecutive pair in $\pi_{j}$ (for some $m \leq j>i$ ). For $i=0$ our claim is true by Lemma 7, hence we can focus on the inductive step. For larger values of $i$, we apply induction to the two copies of $L^{(i-1)}$ : if at least one of the copies has a monochromatic cell or has its monochromatic consecutive pairs at snapshot $\pi_{j}$ (for some $j>i$ ) we are done, since the same property will hold for $L^{(i)}$. The other case occurs when all copies of $L^{(i-1)}$ have their monochromatic consecutive pair at snapshot $\pi_{i}$. Let $\ell_{\nu}, \ell_{\nu}^{\prime}$ be the monochromatic consecutive pair of the $\nu$ th copy of $L^{(i-1)}$ and let $c_{\nu}$ be its color. By the pigeonhole principle, there must be two distinct indices $u, v \leq \nu$ such that $c_{u}=c_{v}$. By property (iii) 
of our construction, the lines $\ell_{u}, \ell_{u}^{\prime}, \ell_{v}, \ell_{v}^{\prime}$ form a quadrilateral in the arrangement of lines of $L^{(i)}$. The quadrilateral will be monochromatic, since by definition the four lines have the same color assigned.

\subsection{Other coloring results}

For the sake of completeness, we end this section by mentioning two issues on coloring vertices or cells instead of lines. We start by considering the chromatic number of $\mathcal{H}_{\text {vertex-cell }}$. Recall that we are interested in colorings in which no hyperedge (containing two or more vertices) is monochromatic.

The following well-known result considers the coloring of cells so that no line is only adjacent to cells of a single color class:

Theorem 8 (Folklore) The chromatic number of $\mathcal{H}_{\text {cell-zone }}$ is 2.

Proof: This claim is equivalent to the fact that the dual graph of the arrangement (where vertices are faces, and there is an edge between two faces if they are adjacent) is bipartite. This result has appeared in recreational texts and concited some research as well [11,14].

We now consider the problem of coloring the vertices of an arrangement so that no cell is monochromatic:

Observation 1 Any arrangement of $n \geq 3$ lines can be colored with at most 3 colors so that the only monochromatic edges of the associated $\mathcal{H}_{\text {vertex-cell }}$ hypergraph are those of size one.

Proof: It is known that the graph obtained from an Euclidean arrangement of lines by taking only the bounded edges of the arrangement has chromatic number 3 [5]: simply sweep with a vertical line from left to right. In this ordering, every vertex in the arrangement is adjacent to at most two predecessors. Thus, we can color the vertices with three colors greedily, and no cell of size at least 2 will be monochromatic.

Naturally, two colors are always necessary, thus the chromatic number will either be 2 or 3 . We note that some arrangements of lines need three colors (for example, any arrangement of three lines), but we have been unable to generalise these examples to arbitrarily large line arrangements.

\section{Guarding Arrangements}

We now consider the vertex cover problem for the hypergraphs defined in Section 2 That is, we would like to select the minimum number of vertices so that any hyperedge is adjacent to the selected subset. Geometrically speaking, we would like to select the minimum number of vertices (or cells or lines), so that each cell (or line or cell, respectively) contains at least one of the selected items. Recall that this problem is the complement of the independent set problem, in the sense that this minimum is equal to the number of vertices minus the size of a largest independent set.

\subsection{Guarding cells with vertices}

We first consider the following problem: Given an arrangement of lines $\mathcal{A}$, how many of the vertices can guard the entire arrangement when lines act as obstacles blocking visibility? This question can be rephrased as finding the smallest subset of vertices $V$ so that each cell contains a vertex in $V$; thus we are looking for bounds on the size of a vertex cover for $\mathcal{H}_{\text {vertex-cell }}$. 
Theorem 9 For any set $L$ of $n$ lines, a vertex cover of the corresponding $\mathcal{H}_{\text {vertex-cell }}$ hypergraph has size at most $n^{2} / 6+n$. Furthermore, $n^{2} / 6$ vertices might be necessary.

Proof: First notice that any arrangement can have at most $2 n$ unbounded cells. Moreover, these cells can be easily guarded with $n$ guards. Thus, we focus our attention to bounded cells. We sweep the arrangement in any fixed direction. Along the sweep we find the vertices of the arrangement one by one. We will color the vertices (with three colors) in the order in which they are discovered.

Vertices that are adjacent to unbounded cells are colored with any arbitrary color among the three possible ones. Now observe that any vertex $v$ that is not adjacent to an unbounded cell must have degree exactly 4. Moreover, when $v$ is encountered, its predecessors $u$ and $w$ have already been colored.

If $u$ and $w$ have distinct colors, we simply assign the third color to $v$. Otherwise, both $u$ and $v$ have the same color assigned. Observe that $v$ is the rightmost vertex of exactly one cell, and in this cell both $u$ and $v$ are present. Since the cell is bounded, it must have another vertex other than $u, v$, and $w$. Moreover, that vertex must have a color different to the color assigned to both $u$ and $v$. Thus, we simply assign the third color to $v$.

With this color assignment, all bounded cells have three vertices with distinct colors on their boundary. In particular, the vertices of any color class can guard all bounded cells. Since we used three colors and the total number of vertices is $\left(\begin{array}{l}n \\ 2\end{array}\right)<n^{2} / 2$, there will be a color class with at most $n^{2} / 6$ vertices.

For the lower bound we use the construction of Füredi et al. [6]. This construction creates a family of arrangements that has $n^{2} / 3$ triangles in which any vertex of the arrangement is incident to at most two of these triangles. In particular, any vertex cover of the triangles will need at least $n^{2} / 6$ vertices.

Recall that the hypergraph $\mathcal{H}_{\text {vertex-cell }}$ has $\left(\begin{array}{l}n \\ 2\end{array}\right)=\frac{n^{2}}{2}-\frac{n}{2}$ vertices. Combining this fact with the preceding bounds on the size of a vertex cover allows us to get similar bounds for the independent set problem:

Lemma 10 For any set $L$ of $n$ lines, a maximum independent set of the corresponding $\mathcal{H}_{\text {vertex-cell }}$ hypergraph has size at least $n^{2} / 3-O(n)$. Furthermore, there exist sets of lines whose largest independent set has size at most $\frac{n^{2}}{3}-\frac{n}{2}$.

\subsection{Guarding lines with cells}

Here we consider the problem of touching all lines of $L$ with a smallest subset of cells, that is, we look for bounds on the size of a vertex cover for $\mathcal{H}_{\text {cell-zone. }}$

We begin with a simple proof that a minimal vertex cover of $\mathcal{H}_{\text {cell-zone }}$ hypergraph has size at most $\left\lceil\frac{n}{2}\right\rceil$, that we will improve below.

Lemma 11 Given a set $L$ of $n$ lines, a minimal vertex cover of the corresponding $\mathcal{H}_{\text {cell-zone }}$ hypergraph has size at most $\left\lceil\frac{n}{2}\right\rceil$.

Proof: We describe a greedy algorithm to find a vertex cover of size $\left\lceil\frac{n}{2}\right\rceil$; we start with an empty set $L$. We find a pair $p, q$ of lines that we still have to cover. Since every two lines cross, there must exist a cell $c$ adjacent to both $p$ and $q$. We add that cell $c$ to the set $L$, and proceed with the unguarded cells. In the last step, if a single line $\ell$ remains to be covered we add to $L$ any cell touching $\ell$. Since each cell (except the last one) of $L$ guards at least two lines, at most $\left\lceil\frac{n}{2}\right\rceil$ cells will be added into $L$.

We next provide a lower bound, and improve as well on the upper bound, for large values of $n$. 
Theorem 12 Given any set $L$ of $n$ lines, a minimal vertex cover of the corresponding $\mathcal{H}_{\text {cell-zone }}$ hypergraph has size at most $\frac{19 n}{48}+o(n)$. Moreover, there exists a set $L$ of $n$ lines, such that every vertex cover of the corresponding $\mathcal{H}_{\text {cell-zone }}$ hypergraph has size at least $\frac{n}{4}$.

Proof: The lower bound is proved by the fact that there exist arrangements where the largest cell has size four (see [13]). This implies that each cell touches at most four lines, and therefore $n / 4$ cells are required to touch them all.

The proof of the upper bound claim is a refined version of the method in Theorem 12 . We first select cells of size four or more, and add them to $L$ until any remaining cell that we add to our set is not guaranteed to cover more than three new lines. We then continue adding cells that cover at least three lines in the same fashion. Finally, we complete our construction with cells that cover two lines as in Lemma 11 .

The first iterations of the algorithm select cells that each cover four new lines. We iteratively select a cell covering four lines as long as the average number of segments of uncovered lines bounding a cell is strictly greater than three. The total number of segments is $n^{2}$, and each contribute to two cells. Every selected cell discards four lines and $4 n$ segments (if the cell is bounded by more than four lines, we only discard exactly four of them, arbitrarily). The total number of cells after the $i$ th iteration is $|C|-i$. By double counting, the number of iterations is the largest value $i$ that satisfies:

$$
\begin{aligned}
\frac{2 n^{2}-8 i n}{|C|-i} & >3, \\
\frac{2 n^{2}-8 i n}{n(n+1) / 2+1-i} & >3, \\
i & \sim \frac{n}{16}+o(n) .
\end{aligned}
$$

Hence we can select roughly $\frac{n}{16}$ cells, covering together $\frac{n}{4}$ lines.

In the second phase of the algorithm, we iteratively select cells covering three new lines. Following the same reasoning, and taking into account the $i \simeq n / 16$ previously selected cells. Using again the double counting argument, we know that the number $j$ of iterations satisfies:

$$
\begin{aligned}
\frac{2 n^{2}-8 i n-6 j n}{|C|-i-j} & >2, \\
\frac{2 n^{2}-n^{2} / 2-6 j n}{n(n+1) / 2+1-n / 16-j} & >2, \\
j & \sim \frac{n}{12}+o(n) .
\end{aligned}
$$

Hence we can select roughly $\frac{n}{12}$ more cells, covering together $\frac{n}{4}$ lines.

Overall, we now have $\frac{n}{16}+\frac{n}{12}+o(n)$ cells covering $\frac{n}{2}$ lines. It remains to cover the remaining $\frac{n}{2}$ lines with $\frac{n}{4}$ cells, each covering two lines, as in Lemma 11 . The total number of cells is therefore

$$
\frac{n}{16}+\frac{n}{12}+\frac{n}{4}+o(n)=\frac{19}{48} n+o(n) .
$$


Corollary 13 For any set $L$ of $n$ lines, a maximum independent set of the corresponding $\mathcal{H}_{\text {cell-zone }}$ hypergraph has size at least $\frac{n^{2}}{2}+\frac{5 n}{48}-o(1)$ and at most $\frac{n^{2}}{2}+\frac{n}{4}+1$.

This Corollary follows directly from the preceding theorem, the fact that the complement of a vertex cover is an independent set, and that any arrangement of $n$ lines in general position has $\frac{n(n+1)}{2}+1$ cells (hence, the $\mathcal{H}_{\text {cell-zone }}$ hypergraph will have that many vertices).

\subsection{Guarding cells with lines}

For the sake of completeness, we also give bounds on the number of lines needed to guard (touch) all cells.

Corollary 14 For any set $L$ of $n$ lines, its minimal vertex cover of the corresponding $\mathcal{H}_{\text {line-cell }}$ hypergraph has size at least $n / 3$ and at most $n+1-\sqrt{n+1}$.

Proof of the lower bound is a direct consequence of the complementarity of the vertex cover/independent set and Theorem 4 (upper bound on the maximum independent set of $\mathcal{H}_{\text {line-cell }}$ ). Analogously, the upper bound is a consequence of Theorem 3 .

\section{Concluding Remarks}

The main open problems arising from our work consist of closing gaps (when they exist) between lower and upper bounds; this is especially interesting in our opinion for the problem of coloring lines without producing any monochromatic cell. We observe that most of our observations hold for pseudo lines as well. Hence, another natural extension would be studying how the bounds change when we consider families of curves any two of which intersect at most $t$ times (for some constant $t>0$ ).

It is also worth noting that there are several computational questions that are interesting as well. For example, it is unclear what the complexity is of deciding whether a given arrangement of lines admits a two-coloring in which no cell is monochromatic.

\section{Acknowledgements}

The authors would like to thank Brian Chan, Michael Hoffmann, and Csaba D. Toth for interesting discussions. We would also like to thank Stefan Felsner for his contribution to the improvement of the constant in Theorem 3 ,

\section{References}

[1] E. Ackerman, and R. Pinchasi. A note on coloring line arrangements. arXiv preprint arXiv:1207.0080, 2012.

[2] P. Bose, H. Everett, and S. Wismath. Properties of Arrangements. International Journal of Computational Geometry, 13(6), pp. 447-462, 2003.

[3] P. Brass, W. Moser and J. Pach. Research Problems in Discrete Geometry. Vieweg Verlag, 2004.

[4] S. Felsner. Geometric Graphs and Arrangements. Springer, Berlin, 2005. 
[5] S. Felsner, F. Hurtado, M. Noy and I. Streinu. Hamiltonicity and colorings of arrangement graphs. Discrete Applied Mathematics, 154(17):2470-2483, 2006.

[6] Z. Füredi and I. Palásti. Arrangements of lines with a large number of triangles. American Mathematical Society, 92(4), 1984.

[7] B. Grünbaum. Arrangements and Spreads. Regional Conf. Ser. Math., American Mathematical Society, 1972 (reprinted 1980).

[8] B. Grünbaum. How many triangles? Geombinatorics, 8:154-159, 1998.

[9] B. Grünbaum. Arrangements of colored lines. Abstract 720-50-5, Notices Amer. Math. Soc., 22(1975), A-200.

[10] B. Grünbaum. Monochromatic intersection points in families of colored lines. Geombinatorics, 9:3-9, 1999 .

[11] B. Grünbaum. Two-coloring the faces of arrangements. Periodica Mathematica Hungarica, 11(3):181-185, 1980.

[12] A. Kaneko and M. Kano. Discrete geometry on red and blue points in the plane - a survey -. In Discrete and Computational Geometry, The Goodman-Pollack Festschrift, Springer, Berlin, B. Aronov, S. Basu, J. Pach, M. Sharir, eds., pp. 551-570, 2003.

[13] J. Leaños, M. Lomelí, C. Merino, G. Salazar, and J. Urrutia. Simple euclidean arrangements with no ( $\geq$ 5)-gons. Discrete and Computational Geometry, 38(3):595-603, 2007.

[14] E. Lucas. Récréations mathématiques IV. Paris, 1894.

[15] T. S. Motzkin. Nonmixed connecting lines. Abstract 67T 605, Notices Amer. Math. Soc., 14(1967), p. 837.

[16] J. O'Rourke. Art Gallery, Theorems and Algorithms. Oxford University Press, 1987.

[17] J. O'Rourke. Visibility. Chapter in Handbook of Discrete and Computational Geometry, CRC Press LLC, Boca Raton, FL, 2nd edition, J. E. Goodman and J. O’Rourke eds., pp. 643-665, 2004 2nd ed.ition).

[18] J. Urrutia. Art Gallery and Illumination Problems. Chapter in Handbook on Computational Geometry, Elsevier Science Publishers, J.R. Sack and J. Urrutia, eds., pp. 973-1026, 2000. 